Published in final edited form as:

Cancer. 2005 December 15; 104(12 Suppl): 2952-2954.

\title{
Quality of Life in Chinese Patients with Breast Cancer
}

\author{
Angela Sun, M.P.H. ${ }^{1}$, Evaon Wong-Kim, Ph.D. ${ }^{2}$, Sarah Stearman, M.P.H. ${ }^{1}$, and Edward A. \\ Chow, M.D. ${ }^{1}$ \\ 1 Chinese Community Health Resource Center, San Francisco, California.
}

2 Department of Social Work, California State University, Hayward, California.

\section{Abstract}

Chinese are the largest Asian group in the U.S., constituting 23.8\% of the nation's total AsianAmerican population. Cancer is the leading cause of death for female Asian Americans, and breast cancer is the most frequently diagnosed cancer among females for all racial/ethic groups in San Francisco, which ranks 4th in the number of Asian Americans and where 152,620 Chinese account for $19.6 \%$ of the city's total population. Previous observations among Chinese immigrant women suggested that a diagnosis of breast cancer may be more detrimental to their well being compared with Chinese women who are born and raised in the U.S. This difference may be due to the lower socioeconomic status, limited English proficiency, poor understanding of the Western medical system among immigrants or other educational or financial considerations. In this qualitative pilot study, the authors sought to increase understanding of the relation between cultural beliefs and quality of life (QOL) among immigrant Chinese women with breast cancer in San Francisco. Specific objectives were 1) to identify these patients' beliefs regarding cancer, life expectancy, and discussion of advance directives; 2) to explore how these beliefs relate to patient QOL; and 3) to generate hypotheses for further study. The overall objective of the pilot study was to investigate questions central to the QOL issue, including what defines QOL for immigrant women and how QOL for them is similar to or different from that for American-born Chinese women.

\section{Keywords}

Asian American Network for Cancer Awareness, Research, and Training; cancer; Asian; Chinese quality of life

\begin{abstract}
According to the Year 2000 Census, Chinese are the largest Asian group in the U.S., constituting 23.8\% of the nation's total Asian-American population. In San Francisco, which is the 13th largest city in the U.S., which ranks 4th in the number of Asian Americans, 152,620 Chinese account for $19.6 \%$ of the city's total population. ${ }^{1}$ Cancer is the leading cause of death for female Asian Americans. In fact, Asian-American females are the first American population to experience cancer as the leading cause of death, ${ }^{2}$ and breast cancer is the most frequently diagnosed cancer among females in the city for all racial/ethnic groups. Among Asian Pacific Island females in the San Francisco Bay Area, the average annual age-adjusted (2000 U.S. population) incidence rate from 1988 to 2001 is 88.2 for invasive breast cancer and 27.1 for in
\end{abstract}

\footnotetext{
Address for reprints: Angela Sun, M.P.H., Chinese Community Health Resource Center, 835 Jackson Street, San Francisco, CA 94133; Fax: (415) 677-2457; E-mail: angelas@ chasf.org.

Presented at Asian American Network for Cancer Awareness, Research, and Training (AANCART): Fifth Asian American Cancer Control Academy, Sacramento, California, October 2004.

Special thanks go to the following individuals for their contribution to the project: Moon S. Chen, Jr., Ph.D., M.P.H.; Carol D'Onofrio, Ph.D.; Reginald C. S. Ho, M.D.; Frederick P. Li, M.D.; Steve McPhee, M.D.; Jeremiah Mock, M.Sc., Ph.D.; and Tung Nguyen, M.D. 
situ breast cancer. ${ }^{3}$ According to the national Surveillance, Epidemiology, and End Results data from 1988 to 1992, the breast cancer rate among Chinese women was 55.0 per 100,000 population. ${ }^{4}$ The breast cancer rate from 1992 to 1996 among Chinese women in the Bay Area was the same as the national rate, with 55 women diagnosed per $100,000 .^{5}$

Previous studies have reported the impact of breast cancer and its treatments on the physical, emotional, psychological, financial, and spiritual aspects of life for multiethnic survivors. 6,7 Nonetheless, it is unclear whether there are changes in quality of life (QOL) among Chinese women who recently immigrated to the U.S. To our knowledge, at the onset of our project, no study had been conducted to examine how breast cancer may jeopardize the QOL of this population. However, observations in working with Chinese immigrant women have suggested that a breast cancer diagnosis may be more detrimental to their well being than for Chinese women who are born and raised in the U.S. This may be due to the lower socioeconomic status and limited English proficiency of immigrants. The lack of educational and financial resources and their lack of understanding of the Western medical system often impose more hardships on the immigrant population.

The objective of the current qualitative pilot study was to increase understanding of the relation between cultural beliefs and QOL among immigrant Chinese women with breast cancer in San Francisco. Specific objectives were 1) to identify these patients' beliefs regarding cancer, life expectancy, and discussion of advance directives; 2) to explore how these beliefs relate to patient QOL; and 3) to generate hypotheses for further study. The objective of the pilot study was to investigate questions central to this issue, including what defines QOL for immigrant women and how QOL for them is similar to or different from American-born Chinese women. This brief report was based on a presentation at the 2004 Asian American Network for Cancer Awareness, Research, and Training conference, where the Principal Investigator summarized preliminary results of the study.

\section{MATERIALS AND METHODS}

To determine whether Chinese immigrants and American-born Chinese women differ in their cancer-related beliefs and QOL, 15 women with breast cancer from each group, for a total sample size of 30 women, were selected for the study. University of Hawaii and University of California-Davis Institutional Review Board approvals were obtained. Chinese women older than age 18 years who had been diagnosed with breast cancer within the past 24 months (at the time of the interview) and were no longer receiving active treatment for the disease were eligible for the study. Healthcare providers from oncology clinics, physician's offices, hospitals, and cancer support groups were contacted to invite their patients to participate in the study. Providers who agreed to assist in recruitment contacted their Chinese patients with breast cancer who were diagnosed less than 2 years previously either by mail or by telephone. Approximately $20 \%$ of the participants were recruited through flyers that were posted at the above-mentioned cancer-related agencies or through advertisements in newspapers. Interested breast cancer patients then called the study telephone number. Telephone screening was administered by a trained, bilingual research assistant who confirmed the callers' eligibility for the study and screened out foreign-born women who had lived in the U.S. for > 15 years. Semistructured, 1.0-1.5 hour, face-to-face interviews were conducted with the 30 participants in either Cantonese or English at a time and location of the participant's choice. The interview included questions related to the participants' experience with breast cancer, cancer-related beliefs, understanding of advance directives, how breast cancer impacted their QOL, and other culturally relevant topics.

Written transcripts of the audiotaped interviews were prepared in the language of the interview. Transcription analysis was completed first manually by the Principal Investigator and the co- 
Principal Investigator working independently. Then, they compared results and resolved any discrepancies, which were identified by referring to the transcription(s) in question and discussing the issues involved. Common themes were identified and are reported below.

\section{RESULTS}

Of the 15 American-born patients, 4 were aged 30-50 years, and 11 were older than age 50 years. Five patients were single, and 10 were married. Seven of the 15 foreign-born patients were aged $30-50$ years, whereas 8 patients were older than age 50 years. Two of the foreignborn patients were single, and 13 were married. Ten of the American-born patients had some college education, whereas 5 had never attended college. Of the foreign-born patients, 1 had a college education, compared with 14 who had not attended college. Of the American-born patients, 2 reported an annual income of $<\$ 35,000$ per year, compared with 11 of the foreignborn patients. Ten of the American-born patients earned $>\$ 35,000$ per year, compared with only 4 of the foreign-born patients. The annual income was unknown for three of the Americanborn patients.

All 15 of the American-born patients had private health insurance, compared with 9 of the foreign-born patients (the remaining 6 patients were insured by Medicaid). Nine of the American-born patients reported $<10$ visits to a Western physician within the past 6 months, and 6 patients reported $>10$ visits. The corresponding numbers among the foreign-born patients were 11 visits and 4 visits, respectively. Conversely, all 15 of the American-born patients reported $<10$ visits to a Chinese physician within the past 6 months, and no patient reported $>10$ visits. Among the foreign-born patients, 6 reported $>10$ visits with a Chinese physician, whereas 9 patients reported $<10$ visits within the past 6 months.

Of the American-born patients, 12 had received radiation therapy, 8 had received chemotherapy, 11 had undergone a lumpectomy, and 4 had undergone a mastectomy. Of the foreign-born patients, 7 patients each had received radiation therapy and chemotherapy, 5 had undergone a lumpectomy, and 8 had undergone a mastectomy. Two of the American-born patients reported receiving psychologic/psychiatric care, whereas only 1 of the foreign-born patients did. The themes identified were 1) sociodemographics (background and life history), 2) beliefs (causes of cancer and the meaning of a breast cancer diagnosis, roles of faith, spirituality, fate, and fatalism), 3) QOL (definitions, influence of cancer diagnosis, and changes in perspective), 4) pain control/alternative medicines (efficacy and side effects of biomedical treatment, use and efficacy of Chinese medicine, and pain control), 5) family (family roles, family support, effects of cancer and its treatment on the family), 6) advance directives (understanding and perceptions), and 7) cultural experiences (living with cancer in the context of culture).

Data analysis suggested that there are important differences between American-born and foreign-born Chinese women in their beliefs about, perceptions of, and experiences with breast cancer. More foreign-born women perceive cancer as a terminal disease. The perception of a cancer diagnosis carrying a stigma existed in both groups; however, it was more prominent in the immigrant group. Both groups of women tended to include family relationships and family support when describing good QOL. American-born Chinese used words such as independence and freedom to describe QOL, and more of them included friendships when describing good QOL. More foreign-born Chinese included wealth as an important dimension of QOL, and many of these women saw breast cancer as another traumatic event that compounded the difficulties brought about by immigration. Stressful life in the U.S. also was perceived as a possible cause of their breast cancer diagnosis. These beliefs, perceptions, and experiences may have important implications for cancer support services and survivorship. Both groups of women described their breast cancer diagnosis as a wake-up call, which reminded them to take 
better care of themselves. Both groups of women identified cancer support groups as a way to help them cope with cancer. However, both groups identified a need for additional supportive services, especially in-home support services.

\section{DISCUSSION}

Due to budgetary constraints, the current sample size was relatively small. Nonetheless, the demographics of the 30 participants resembled closely those of Chinese-American women with breast cancer in the San Francisco Bay Area. Data from this study were collected from individuals who responded to the invitation to participate, and these individuals may have characteristics and experiences different from nonrespondents. Although they are not necessarily representative of all Chinese-American patients with breast cancer, the findings from this exploratory study enhanced our understanding of the influence of culture on individual patient's perceived QOL.

To our knowledge, the current study was one of the first to examine QOL among Chinese Americans with breast cancer. Data collected will be valuable for the development and deliverance of culture-specific services for both immigrant and American-born Chinese. In a forthcoming article, results from this study will be discussed in further detail. Future studies in other ethnic populations will enhance the quality of care for all cancer patients in the U.S..

\section{References}

1. Bureau of the Census. Statistical abstract of the United States. [accessed January 10, 2005]. Available from URL: http://www.census.gov/prod/2001pubs/statab/sec01.pdf

2. Asian American Network for Cancer Awareness, Research, and Training - 2005. [accessed January 10, 2005]. Available from URL: http://www.aancart.org/About\%20us.htm

3. Le, GM.; O’Malley, CD., et al. Cancer Incidence and Mortality in the Greater Bay Area, 1988-2001. Union City, CA: Northern California Cancer Center; 2004.

4. Miller, BA.; Kolonel, LN.; Bernstein, L., et al., editors. Racial/ethnic patterns of cancer in the United States 1988-1992. Bethesda, MD: National Cancer Institute; 1996. NIH Pub. No. 96-4104

5. Glanz, K. Cancer in women of color monograph. Bethesda, MD: National Cancer Institute, Department of Health and Human Services; 2003.

6. Ashing-Giwa KT, Padilla G, Tejero J, et al. Understanding the breast cancer experience of women: a qualitative study of African American, Asian American, Latina and Caucasian cancer survivors. Psychooncology 2004;13:408-428. [PubMed: 15188447]

7. Kagawa-Singer M, Wellisch D, Durvasula R. Impact of breast cancer on Asian American and Anglo American women. Cult Med Psychiatry 1997;21:449-480. [PubMed: 9492974] 